\title{
KPFM surface photovoltage measurement and numerical simulation
}

\author{
Clément Marchat ${ }^{1,2,}{ }^{*}$, James P. Connolly ${ }^{2}$, Jean-Paul Kleider ${ }^{2}$, José Alvarez², Lejo J. Koduvelikulathu ${ }^{3}$, \\ and Jean Baptiste Puel ${ }^{1,4}$ \\ ${ }^{1}$ IPVF, Institut Photovoltaïque d'Île-de-France, 30 route Départementale 128, 91120 Palaiseau, France \\ 2 GeePs, UMR CNRS 8507, Centralesupélec, Université Paris-Sud, Université Paris-Saclay, Sorbonne Université, \\ 11 rue Joliot Curie, Plateau de Moulon, 91192 Gif sur Yvette, France \\ 3 ISC-Konstanz e.V., Rudolf-Diesel-Straße 15, 78467 Konstanz, Germany \\ ${ }^{4}$ EDF R\&D, 30 Route Départementale 128, 91120 Palaiseau, France
}

Received: 13 November 2018 / Received in final form: 26 March 2019 / Accepted: 29 April 2019

\begin{abstract}
A method for the analysis of Kelvin probe force microscopy (KPFM) characterization of semiconductor devices is presented. It enables evaluation of the influence of defective surface layers. The model is validated by analysing experimental KPFM measurements on crystalline silicon samples of contact potential difference $\left(\mathrm{V}_{\mathrm{CPD}}\right)$ in the dark and under illumination, and hence the surface photovoltage (SPV). It is shown that the model phenomenologically explains the observed KPFM measurements. It reproduces the magnitude of SPV characterization as a function of incident light power in terms of a defect density assuming Gaussian defect distribution in the semiconductor bandgap. This allows an estimation of defect densities in surface layers of semiconductors and therefore increased exploitation of KPFM data.
\end{abstract}

Keywords: KPFM / SPV / surface defects / modeling and band bending

\section{Introduction}

Kelvin Probe Force Microscopy (KPFM, Fig. 1) is a scanning probe microscopy technique. It is a combination of the Kelvin probe and of Atomic Force Microscopy methods [1]. The technique consists in evaluating the difference in work function between two conducting materials, by using a nanometer scale tip (the "KPFM tip"), and placing it close to the material to be characterised, where a difference in work function leads to an electrostatic force developing between the two, which is translated as an oscillation of the tip's cantilever. A bias applied via an external circuit is varied until the force and hence the electrostatic field between sample and KPFM tip is cancelled. There are a number of modes of operation as discussed in the original Rosenwaks paper [1] and subsequent work [2].

The analysis of the technique requires consideration of a range of parameters [3] which, influence the work function of the semiconductor under consideration, given well characterised AFM tips. The parameters which determine the surface work function of the semiconductor sample are

\footnotetext{
* e-mail: clement.marchat@ipvf.fr
}

in short those which determine the Fermi level at the surface starting with a good knowledge of the electronic band structure of the semiconductor in question.

The list of parameters contains minority and majority carrier transport parameters including diffusion constant and carrier lifetimes. Contributing to these transport parameters are the full range of relevant recombination mechanisms which in this study on silicon samples are dominated by Auger and Shockley-Read-Hall recombination.

Parameters included in this work are first doping levels determining equilibrium majority carrier densities. We also include surface and bulk defects with energies in the forbidden gap described by donor-like and acceptor-like Gaussian distributions.

The KPFM technique allows evaluation of a range of transport and electrostatic parameters of semiconductor structures on the local scale of the order of tens of nanometers. This includes evaluation of transport parameters and of band bending in homogeneous and heterogeneous materials by performing scans as a function of position. The characterization in the dark $\left(\mathrm{V}_{\mathrm{CPD}}\right.$ - dark $)$ and under light $\left(\mathrm{V}_{\mathrm{CPD}}\right.$ - light $)$ enables evaluation of the surface photovoltage (SPV defined as the difference between $\mathrm{V}_{\mathrm{CPD} \text { - light }}$ and $\mathrm{V}_{\mathrm{CPD} \text { - dark }}[4]$ ) which can be used to evaluate the potential open circuit voltage of semiconductor structures [5]. 


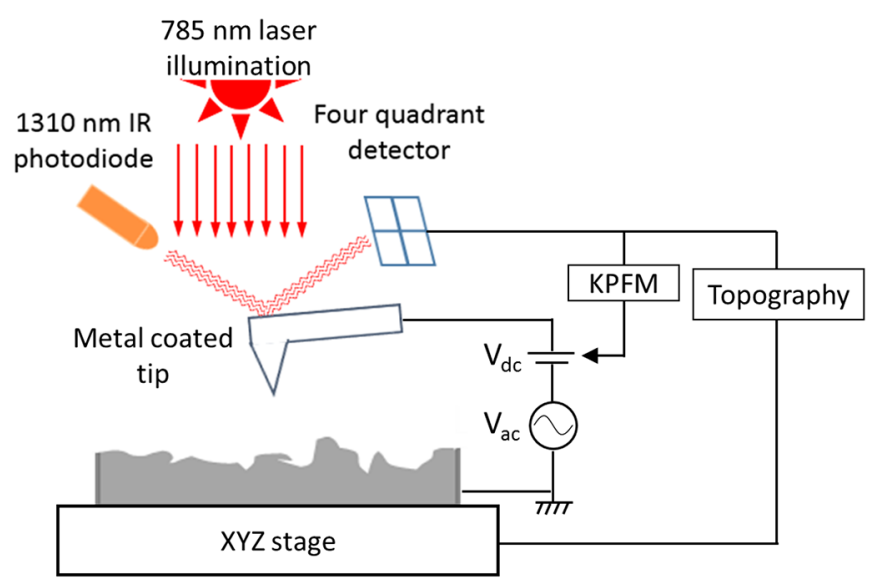

Fig. 1. Kelvin probe force microscopy setup schematic. The conducting cantilever carrying the KPFM tip is scanned over a surface while $\mathrm{AC}+\mathrm{DC}$ potential is applied. The $\mathrm{AC}$ signal is a sinusoid whose frequency matches the mechanical resonance of the cantilever. The four-quadrant detector provides feedback in order to minimise cantilever oscillation by varying the DC signal thereby yielding the sample work function compared to the tip one.

The SPV signal is determined by the parameters mentioned above [3] which can be summarised as being the material band structure, transport parameters, and defect properties first and foremost. This is for SPV compounded by the requirement of a knowledge of absorption and photogeneration as a function of position, and solution of semiconductor transport, continuity and Poisson equations to yield the carrier density as a function of position, and hence the spatial variation of the quasi-Fermi levels of electrons and holes.

The solution of transport equations is performed by numerical software [6] which also provides a library of materials electronic transport and band structure parameters.

We conclude that the analysis of KPFM data including $\mathrm{V}_{\mathrm{CPD}}$ and SPV requires a wide range of parameters which are relatively well known except for the defect distributions. This work is therefore focused on the comparison between simulation of SPV depending of defect distribution properties and experimental measurements to correlate them and extract estimation of the experimental surface defect properties.

Concerning the numerical modelling, this work has extensively developed recent one dimensional KPFM modelling work [7] to enable the evaluation of the influence of surface defects. This development has generalised the 1D approach to two dimensions in order to be able to take location of electrodes on the sample into account, and also perform AFM scans over the surface of semiconductor samples. This allows the evaluation of semiconductor doping and compositional junctions. It has also developed a complex description of defects of acceptor and donor types, with Gaussian and tail distributions of variable peak, width, and characteristic energy.

This has been further developed as a separate software tool KELSCAN acting as an interface to SILVACO which enables parameter scans over a wide range of parameters.
These include scans varying doping levels, defect density properties, illumination spectrum (including monochromatic sources) and illumination power.

Using this experimental characterisation and theoretical analysis, the aim of this work is to enable the full use of the rich set of information which may be yielded by KPFM characterization for use in opto-electronic device characterization, and in particular in the study of photovoltaic solar cells.

\section{Methods}

\subsection{Experimental}

The samples studied here are n-type and p-type monocrystalline (111) silicon wafers of thickness above $500 \mu \mathrm{m}$, (doping levels $\mathrm{N}_{\mathrm{D}}$ and $\mathrm{N}_{\mathrm{A}}$ both equal to $10^{17} \mathrm{~cm}^{-3}$ ) which only absorb photons of energy greater than $\approx 1.12 \mathrm{eV}$ corresponding to wavelengths below $1100 \mathrm{~nm}$. Both the n-type and p-type samples surface were characterized as received without surface treatment. A stable native oxide layer is expected at their surface.

Each sample was characterised under stable ambient conditions of temperature and humidity during its whole measurement. The samples being exempt of any internal junction and having sufficient thickness to totally absorb over gap illumination then only the front surface of the samples can be the source for a SPV signal under illumination.

The characterization was performed with a TRIOS AFM platform allowing light and dark measurements (Fig. 1). This AFM uses a $1310 \mathrm{~nm}$ wavelength laser for its Tip Deflexion Detection System (TDDS) and feedback loop which enables the KPFM tip to be maintained at a constant distance from the surface [1]. This corresponds to a photon energy $(0.976 \mathrm{eV})$ well below the Si absorption edge. This light being not absorbed in the $\mathrm{Si}$ does not produce any SPV signal allowing reliable $V_{\mathrm{CPD}}$ - dark values to be measured. Furthermore a verification of the effect of this laser was done by changing its position on the tip's cantilever and so the distance to the measured area. For all positions, either just above the tip or near the base of the cantilever, the observed CPD values variations were within the experimental noise.

KPFM measurements were performed using ARROWEFM conductive tips with a PtIr coating. The tip work function didn't require calibration because only SPV measurement were performed and studied. Measurements were performed in the KPFM amplitude modulation (AM) mode rather than the frequency modulation (FM) one [1,2]. The AM mode was chosen because lateral resolution was not a problem on the homogeneous bulk samples studied, allowing focus on the superior surface potential resolution that can be achieved with the AM mode.

The $\mathrm{V}_{\mathrm{CPD}}$ - light values were obtained using a $785 \mathrm{~nm}$ laser of variable intensity. This is absorbed in the Si sample and leads to a modification of carrier concentrations and distributions which is at the origin of the SPV signal.

The laser intensity has been adjusted by both varying the nominal power of the $785 \mathrm{~nm}$ laser that ranges from $5 \mathrm{~mW}$ to $200 \mathrm{~mW}$ and the spot diameter which could be 
varied between $240 \mu \mathrm{m}$ and $5 \mathrm{~mm}$. The determination at the position of the sample of the incident power for each nominal power value and the knowledge of the laser spot diameter allowed to obtain the incident laser power density. Those conditions allowed to have two ranges of power density, which combined yielded the full range of three to four orders of magnitude in laser power density. The illuminated area diameter was always above the photo carrier diffusion length of $80 \mu \mathrm{m}$ calculated assuming a bulk lifetime value of $20 \mu \mathrm{s}$ and a hole mobility of $12 \mathrm{~cm}^{2} / \mathrm{s}$ obtain from semiconductors properties archive (see ioffe). This allows to have a generation volume above the carrier diffusion volume.

The SPV measurements with the TRIOS KPFM setup were performed for this range of illumination intensities over four orders of magnitude going from tens of $\mathrm{W} / \mathrm{m}^{2}$ to over $10^{5} \mathrm{~W} / \mathrm{m}^{2}$.

\subsection{Modelling}

The simulations were performed using in-house software KELSCAN developed by the IPVF institute, and which delivers a targeted KPFM modelling tool using SILVACO software [6]. The model uses numerical solutions of semiconductor transport equations coupled with Poisson's equation and includes standard radiative and nonradiative (Auger and Shockley-Read-Hall) recombination mechanisms. These aspects have been described in detail in previous work of one dimensional Silvaco KPFM modelling by Huang et al. [7].

The KELSCAN solution extends previous work in two directions. The first is a two dimensional definition of samples which allows a realistic representation of charge distribution within the volume of the device, particularly from the perspective of placement of electrodes on the sides of the samples (orthogonal to the KPFM tip) or at the lower surface of the sample (below the KPFM tip). This methodology furthermore allows the specification of arbitrary doping and compositions profiles in the sample and enables the study of doping gradients and interfaces, and of $p n$ junction devices in cross section. In this two dimensional modelling, simulation of KPFM tip scanning across the surface is possible, and a more exact simulation of the consequences of tip geometry and sample contact placement is enabled.

The second and most important development is the inclusion of bulk donor and acceptor type defects. These are specified by peak donor or acceptor defect density, Gaussian defect distribution width and energy position.

The contact potential voltage $\mathrm{V}_{\mathrm{CPD}}$ is modelled by physically defining a $2 \mathrm{D}$ probe of realistic dimensions with a workfunction of $5 \mathrm{eV}$ and placing it at the experimental distance (typically $100 \mathrm{~nm}$ ) from the sample surface. An air ambient is defined between the sample and the tip as in the experimental setup.

A numerical interpolation scheme in KELSCAN evaluates the zero field condition by comparing fields with no bias applied between KPFM tip and sample contacts, and with non-zero bias estimated from the analytical value of $\mathrm{V}_{\mathrm{CPD}}[3]$ which can be easily estimated from the tip and sample material work functions and the doping level.
KELSCAN also provides a range of ten parameter scanning functionalities allowing scanning calculations of dark or light $\mathrm{V}_{\mathrm{CPD}}$, or $\mathrm{SPV}$, as a function of these parameters. The ten parameters which we will not list for brevity include:

- acceptor and donor-type band tail and Gaussian distributions: Width, peak energy, peak density;

- defect layer thickness: the physical extent of the defect layer below the sample surface;

- incident light intensity and wavelength.

The underlying physics of the problem has been detailed by Huang [7] and references therein, while this work extends this from $1 \mathrm{D}$ to $2 \mathrm{D}$ and includes critical surface defect densities. We only note here that the numerical method solves the continuity equations, drift and diffusion, and Poisson equations together with geometric optics for light absorption of illuminating spectra.

\section{Results and discussion}

\subsection{SPV modelling method}

This study evaluates the SPV dependence on the dominant parameters in the c-Si samples. These parameters are the defect state distribution parameters we have seen earlier, including the defect layer thickness. The modelling methodology is focused on validating KPFM simulation as a means to analysing experimental data on c-Si wafer at ambient condition. This section details the procedure for simulating SPV characterisation on the n-type samples for a single illumination power. A similar procedure is followed for the p-type sample but is not given in detail for the sake of brevity.

\subsubsection{Defect layer thickness}

We first address the matter of the influence of defect layer thickness. We assume a surface layer with a finite defect layer thickness extending over the entire surface. The defects have a single Gaussian distribution in energy. The charge of ionised defects is assumed of opposite sign to ionised dopants in the bulk of the semiconductor. We therefore specify defect layer consisting only of acceptortype defects for the n-type sample (donor-type defects for the p-type sample).

We first study the dependence of the SPV signal as a function of defect thickness for a fixed defect density, as shown in Figure 2a. The defect density chosen here is the limiting case of very high acceptor defect density at $10^{19} \mathrm{~cm}^{-3}$. This defines an interface between dissimilar charges similar to a standard $p n$ junction, and with a corresponding space-charge region. For very high defect densities as used here and for thinner layers, the result is a completely depleted surface layer and corresponding surface field. The stronger this surface field, the narrower the space-charge region, with the consequence that ionised defect layers at the limit of high doping show decreasing field strengths as their thickness is increased. This phenomenon is visible in Figure 2a, with the decreasing SPV signal as the defect layer thickness is increased. It reaches a limit when the surface is no longer ionised. 

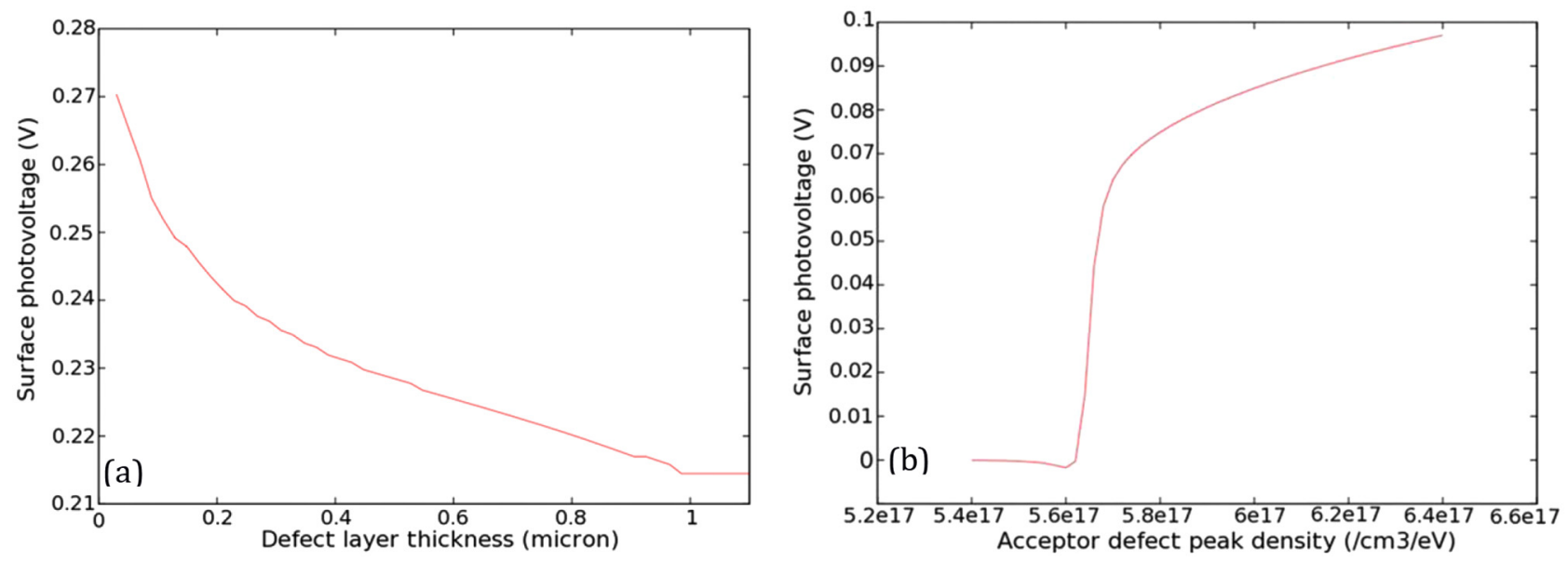

Fig. 2. Surface photovoltage simulated $\left(785 \mathrm{~nm}\right.$ illumination $\left.1000 \mathrm{~W} / \mathrm{m}^{2}\right)$ on bulk silicon doped $\mathrm{n}$-type at $\mathrm{N}_{\mathrm{D}}=10^{17} \mathrm{~cm}^{-3}(\mathrm{a})$ as a function of the thickness of the surface defect layer showing a reduction to a constant value for thick charge neutral layer and (b) as a function of acceptor defect density $\mathrm{N}_{\mathrm{DA}}$ (for defective layer thickness $0.1 \mu \mathrm{m}$ ) showing an initial reduction in SPV noted as a slight reduction in effective n-type doping, before $\mathrm{N}_{\mathrm{DA}}$ significantly larger than $\mathrm{N}_{\mathrm{D}}$ leads to the formation of an effective pn junction.

In this case of a thick defect layer with a space-charge that don't reach the neutral front layer, the Fermi level is naturally insensitive to illumination at moderate illumination levels (where we mean illumination levels analogous to low injection, where the photogenerated minority carrier density remains low compared with the ionised acceptorlike defect densities).

The conclusion of this brief study of defect layer thickness is that this free parameter must be used in order to evaluate surface density. Nevertheless, in the absence of characterization of this parameter, we rely on qualitative indications from the literature [8] assuming that defect thicknesses below $1 \mu \mathrm{m}$ are representative. The following studies therefore choose a defect layer thickness range of $0.1 \mu \mathrm{m}$ to $1 \mu \mathrm{m}$ as limit for this parameter.

\subsubsection{Acceptor type defect peak density}

Having established a proposed value for the defect layer thickness, we consider the defect distribution. While complex trap models are available, we assume for simplicity that defect densities can be represented by Gaussian distributions. We fix a Gaussian distribution width of $0.1 \mathrm{eV}$ again qualitatively in line with values reported in the literature. We choose to use the peak acceptor type defect density $\mathrm{N}_{\mathrm{DA}}$.

Figure $2 b$ shows SPV simulation as a function of $\mathrm{N}_{\mathrm{DA}}$ for the n-type silicon bulk sample and peak position of the Gaussian defect distribution at $0.5 \mathrm{eV}$ above the valence band maximum. The illumination used was set at a low level of $1000 \mathrm{~W} / \mathrm{m}^{2}$, which can be obtained experimentally by defocused light beam.

We can observe that for acceptor defect densities below the intentional doping level of $10^{17} \mathrm{~cm}^{-3}$, there is no appreciable SPV signal. This means that illumination is not appreciably changing the position of the quasi Fermi level for majority carrier and hence the apparent work function in this material.
A transition is observed at defect densities of the same order of the intentional doping level. After a slight decrease for $\mathrm{N}_{\mathrm{DA}}$ just below the intentional doping level, the SPV signal increases markedly, corresponding to a hole quasi Fermi level moving rapidly towards the valence band. The $\mathrm{N}_{\text {DA }}$ parameter scan reaches the experimentally measured value of $\mathrm{SPV}=80 \mathrm{mV}$ at a peak density of $5.9 \times 10^{17} / \mathrm{cm}^{2}$.

With this method, we are therefore about to fit experimental values of KPFM measurements and estimate the density of defects subject to the assumptions mentioned previously. The method can be expanded by the KELSCAN tool exploring the larger parameter set determining the defect properties of materials as mentioned in the methodology section.

\subsection{SPV as a function of illumination intensity}

Following the definition of the simulation methodology using the low-light intensity limit, we now extend to the full range of measurements starting with SPV measurements on the n-type Si sample. This full range of SPV measurements (Fig. 3) show experimental data at both low and high incident light intensity achieved by using laser beam spot diameter conditions of 240 microns (Figs. 3a and 3b) and then of $2 \mathrm{~mm}$ (Fig. 3a) or $5 \mathrm{~mm}$ (Fig. 3b).

A good overlap with intensity data between those two optical settings of the setup is observed. Some variation in the data is nevertheless visible, which is consistent with the noise from the experimental measurement of $10 \mathrm{mV}$ for the measured potential and around $5-15 \%$ for light power density. The obtained curves show a logarithmic dependence of SPV on incident light power. This shows a slope close to $\pm \mathrm{kT} / \mathrm{q}$, the sign depending on the defect type.

The modelling explores the SPV by varying the defect distribution (acceptor type defects for the n-type sample, donor type defects for the p-type $\mathrm{Si}$ ). While a detailed description of the procedure is beyond the scope of this 

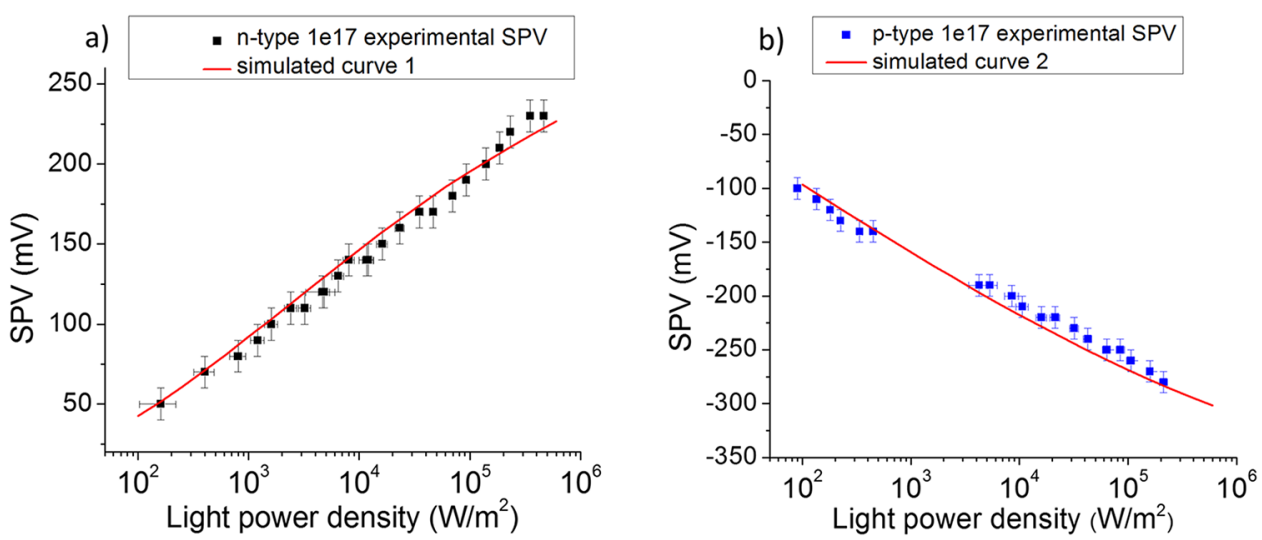

Fig. 3. Measured and simulated surface photovoltage on crystalline (111) silicon doped (a) n-type $\mathrm{NA}=10^{17} \mathrm{~cm}^{-3}$ and (b) p-type $\mathrm{ND}=10^{17} \mathrm{~cm}^{-3}$. A good match between focused and defocused illumination is achieved. Modelling shows a good match with the experimental data in both case.

Table 1. Surface defect parameters extracted from the simulations used in Figure 3.

\begin{tabular}{lll}
\hline Parameters & Sample n-type $10^{17} \mathrm{~cm}^{-3}$ & Sample p-type $10^{17} \mathrm{~cm}^{-3}$ \\
\hline Energetic distribution of surface states $\left(\mathrm{cm}^{-2} \mathrm{eV}^{-1}\right)$ & $6 \times 10^{13}$ & $7 \times 10^{13}$ \\
Gaussian distribution width (eV) & 0.1 & 0.1 \\
Characteristic energy (eV/valence band) & 0.56 & 0.56 \\
\hline
\end{tabular}

paper, the main result of these investigations is that the observed SPV dependence close to $\pm \mathrm{kT} / \mathrm{q}$ is indicative of very high defect densities in surface defect layer, where "very high" means significantly greater than the intentional doping of opposite type.

A good phenomenological agreement is obtained between measured and modelled SPV as a function of incident light power. The parameters extracted from the simulation can be seen in Table 1. Qualitative values of defects peak density, energy width, energy position and defect layer thickness can be obtained from the simulation parameters used. From the literature the surface band bending can be linked to the parameter of energetic distributions of interface states $D_{\mathrm{it}}(E)$ [9]. This parameter, which in our case is an energetic distribution of surface states $\left(D_{\mathrm{st}}(E)\right)$, can be compared to the combination of the defect peak density and defect layer thickness parameters used in the simulation. The value of $D_{\mathrm{st}}(E)$ calculated through the parameters extracted from the simulation are in agreement with the values found in the literature for $\mathrm{Si}$ surface with native oxide [9].

\section{Conclusions}

This paper has presented a characterization method for KPFM measurement and subsequent analysis. To these ends, KPFM measurements of simple crystalline $\mathrm{Si}$ samples were presented and analysed, demonstrating the development of methodology of understanding KPFM measurements. The challenges of interpreting these measurements is clear from the wide range of parameters involved. We have investigated a restricted set of parameters and chosen the defect layer thickness and the peak defect density in order to illustrate the statements that can be made concerning KPFM measurements under illumination and in the dark.

The simulation allows us to shed light on the physical impacts of defect densities on the electronic transport properties and band structure of materials. The KPFM experimental SPV measurements depending of illumination density were reproduced by simulation for both n-type and p-type samples. A qualitative indication of defect properties at the surface of the samples has been extracted from the parameters of the scanning simulation method.

The authors are grateful for financial support of the COST Action MP1406 "MultiscaleSolar" enabling exchanges and the support from the French Government via the program of investment for the future (Programme d'Investissement d'Avenir - ANR-IEED002-01).

\section{Author contribution statement}

Clement Marchat is the principle author and James P. Connolly contributed substantially to the writing. Jean-Paul Kleider and Jose Alvarez coordinated the manuscript preparation and submission. Lejo J. Koduvelikulathu and Jean Baptiste Puel contributed with input on experimental and theoretical issues. 


\section{References}

1. P.Y. Rosenwaks, S. Saraf, O. Tal, A. Schwarzman, D.T. Glatzel, P.D.M.C. Lux-Steiner, Kelvin probe force microscopy of semiconductors, in Scanning Probe Microscopy, edited by S. Kalinin, A. Gruverman (Springer, New York, 2007), pp. 663-689

2. P. Narchi et al., Cross-sectional investigations on epitaxial silicon solar cells by kelvin and conducting probe atomic force microscopy: effect of illumination, Nanosc. Res. Lett. 11, 55 (2016)

3. L. Kronik et al., Surface photovoltage spectroscopy of semiconductor structures: at the crossroads of physics, chemistry and electrical engineering, Surf. Interface Anal. 31, 954 (2001)

4. V. Donchev et al., Surface photovoltage phase spectroscopy - a handy tool for characterisation of bulk semiconductors and nanostructures, Mater. Sci. Eng. B 129, 186 (2006)
5. V. Donchev et al., A surface photovoltage spectroscopy system used for minority carrier diffusion length measurements on floating zone silicon, J. Optoelectr. Adv. Mater. 7, $533(2015)$

6. Silvaco Inc., ATLAS user's manual (2012). Available at http://silvaco.com

7. Y. Huang et al., A new approach to modelling Kelvin probe force microscopy of hetero-structures in the dark and under illumination, Opt. Quant. Electr. 50, 41 (2018)

8. R.C. Newman, Defects in silicon, Rep. Prog. Phys. 45, 1163 (1982)

9. B. Stegemann et al., Passivation of Crystalline Silicon Wafers by Ultrathin Oxide Layers: Comparison of Wetchemical, Plasma and Thermal Oxidation Techniques, in IEEE 7th World Conference on Photovoltaic Energy Conversion (2018)

Cite this article as: Clément Marchat, James P. Connolly, Jean-Paul Kleider, José Alvarez, Lejo J. Koduvelikulathu, Jean Baptiste Puel, KPFM surface photovoltage measurement and numerical simulation, EPJ Photovoltaics 10, 3 (2019) 